Enrique Piacentini

Marc Wysocki

Lluis Blanch

\section{Erratum to: A new automated method versus continuous positive airway pressure method for measuring pressure-volume curves in patients with acute lung injury}

Published online: 2 September 2010

(C) Copyright jointly held by Springer and ESICM 2010

The online version of the original article can be found under doi:10.1007/s00134-008-1322-2.

\section{E. Piacentini}

Doctorando UAB, Intensive Care Unit,

Hospital Mútua de Terrassa, Terrassa, Spain

\section{Wysocki}

Medical Research, Hamilton Medical AG,

Bonaduz, Switzerland

L. Blanch (-

CIBER Enfermedades Respiratorias, Critical Care Center,

Hospital de Sabadell, Corporació Parc Taulí,

Institut Universitari Fundació Parc Taulí,

Universitat Autónoma de Barcelona,

Parc Taulí s/n, 08208 Sabadell, Spain

e-mail: lblanch@tauli.cat

Tel.: +34-93-7458323

Fax: +34-93-7233863
Erratum to: Intensive Care Med (2009) 35:565-570 DOI 10.1007/s00134-008-1322-2

At the time of publication the author E. Piacentini was a $\mathrm{PhD}$ student of the Universitat Autónoma de Barcelona, and this should have been reflected in his affiliation. The correct affiliation is as follows:

\author{
E. Piacentini \\ Doctorando UAB, \\ Intensive Care Unit, \\ Hospital Mútua de Terrassa, \\ Terrassa, Spain
}

